\title{
DIGITALCOMMONS
}

@WAYNESTATE —

Wayne State University

$10-1-2004$

\section{Variational Stability and Marginal Functions via Generalized Differentiation}

Boris S. Mordukhovich

Wayne State University, boris@math.wayne.edu

Nguyen Mau Nam

Wayne State University

\section{Recommended Citation}

Mordukhovich, Boris S. and Nam, Nguyen Mau, "Variational Stability and Marginal Functions via Generalized Differentiation" (2004). Mathematics Research Reports. Paper 27.

http://digitalcommons.wayne.edu/math_reports/27

This Technical Report is brought to you for free and open access by the Mathematics at DigitalCommons@WayneState. It has been accepted for inclusion in Mathematics Research Reports by an authorized administrator of DigitalCommons@WayneState. 


\title{
VARIATIONAL STABILITY AND MARGINAL FUNCTIONS VIA GENERALIZED DIFFERENTIATION
}

\author{
BORIS S. MORDUKHOVICH and NGUYEN MAU NAM
}

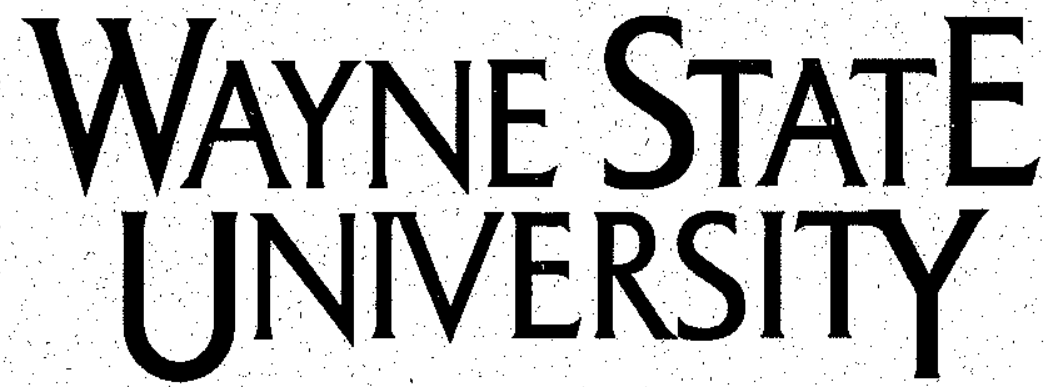

Detroit, MI 48202

Department of Mathematics

Research Report

2004 Series

$\# 10$

This research was partly supported by the National Science foundation and the Australian Research Council. 


\title{
VARIATIONAL STABILITY AND MARGINAL FUNCTIONS VIA GENERALIZED DIFFERENTIATION
}

\author{
BORIS S. MORDUKHOVICH \\ Department of Mathematics, Wayne State University \\ Detroit. MI 48202, U.S.A., boris@math.wayne.edu \\ NGUYEN MAU NAM \\ Department of Mathematics, Wayne State University \\ Detroit. MI 48202. U.S.A., nam@math.wayne.edu
}

Robust Lipschitzian properties of set-valued mappings and marginal functions play a crucial role in many aspects of variational analysis and its applications, especially for issues related to variational stability and optimization. We develop an approach to variational stability based on generalized differentiation. The principal achievements of this paper include new results on coderivative calculus for set-valued mappings and singular subdifferentials of marginal functions in infinite dinnensions with their extended applications to Lipschitzian stability. In this way we derive efficient conditions ensuring the preservation of Lipschitzian and related properties for set-valued mappings under various operations, with the exact bound/modulus estimates, as well as new sufficient conditions for the Lipschitz continuity of marginal functions.

Key words: optimization and variational, robust stability and sensitivity, marginal and value functions, generalized differentiation

MDC2000 subject classification: Primary: 90C30, 49J52

\section{Introduction}

Variational analysis has been well recognized as a fruitful area in applied mathematics dealing, first of all, with optimization-related issues while also applying variational principles and approaches to a large spectrum of problems, which may not be of a variational nature. We refer the reader to the books by Rockafellar and Wets [30], Borwein and Zhu [3], and Mordukhovich [18] for the key developments on basic theory of variational analysis and its numerous applications including those to various topics in operations research. Since nonsmooth functions, sets with nonsmooth boundaries, and set-valued mappings naturally and frequently arise in optimization-related problems and variational techniques, the qualitative and quantitative aspects of generalized differentiation lie at the very heart of variational analysis and its applications.

This paper mainly concerns developing a generalized differential approach to variational stability, which is understood here from the viewpoint of robust Lipschitzian behavior of set-valued mappings and marginal functions preserved under perturbations of the initial data. Indeed, such a robust Lipschitzian stability plays a crucial role in many aspects of variational analysis and optimization, especially those related to sensitivity of feasible and optimal solution sets under parameter perturbations; see the afore-mentioned books and 
also the ones by Bonnans and Shapiro [2] and by Facchinei and Pang [6] with the extended bibliographies therein.

The main single tool of our analysis is the construction of coderivative (adjoint derivative) for set-valued mappings introduced by Mordukhovich [13] and then developed and applied in many publications; see, e.g., Borwein and Zhu [3], Dontchev, Lewis and Rockafellar [5], Ioffe [7], Ioffe and Penot [8], Jourani and Thibault [9, 10], Levy and Mordukhovich [11], Mordukhovich [1 1. 15, 16, 17, 18], Mordukhovich and Shao [21, 22, 23], Outrata [25], Penot [26], Rockafellar and Wets [30], Thibault [32], Treiman [33], Ye and Ye [35], Ye and Zhu [36], and the references therein.

It is well known (se' Mordukhovich [14] and Rockafellar and Wets [30]) that robust Lipschitzian behavior of set-valued mappings $F: X \rightrightarrows Y$ between finite-dimensional spaces $X$ and $Y$ can be completely characterized by using the normal coderivative of $F$ at the reference graph point $(\bar{x}, \bar{y}) \in$ gph $F$ defined by

$$
D_{N}^{*} F(\bar{x}, \bar{y})\left(y^{*}\right):=\left\{x^{*} \in X^{*} \mid\left(x^{*},-y^{*}\right) \in N((\bar{x}, \bar{y}) ; \operatorname{gph} F)\right\}
$$

via the nonconvex normal cone intruduced by Mordukhovich [12]. In infinite-dimensional spaces $X$ and $Y$, coderivative constructions of type (1.1) do not provide anymore characterizations of Lipschitzian stability. Such characterizations of the classical Lipschitz continuity and its proper set-ralued counterpart. known as the Aubin "pseudo-Lipschitzian" (or Lipschitz-like) property: were established in Mordukhovich [16] and Mordukhovich and Shao [23] involving the so-called mircd codernutive $D_{M}^{*} F(\bar{x}, \bar{y})$, which is generally different from (1.1) in infinite dimensions: se' Section 2. Moreover, the usage of both normal and mixed coderivatives provides tuo-suded cstmmates of the exact bounds for Lipschitzian moduli, which give precise formulas to compute the exact bounds under certain coderivative normality conditions; see Mordukhovich [17, 18].

In this paper we develop new results of coderivative calculus that, being combined wish the afore-mentioned characterizations, allow us to ensure the preservation of robust Lipschitzian stability under various compositions of set-alued mappings, together with relationships between the corresponding exact Lipschitzian bounds. As consequences of these results, we derive efficient conditions for preserving the related metric regularity and openness/covering properties of sct-valued mipplings under compositions, with relationships between their exact bounds.

The usage of mixed coderivatives allows us to estallish also new upper estimates for singular subgradients of the so-called margmal functions of the type

$$
\mu(x):=\inf _{y=F(x !} F(x, y)
$$

generated by a set-valued mapping $F: X=Y$ between Banach spaces and an extendedreal-valued function $\varphi: X \times Y \rightarrow \overline{\mathbb{R}}:=(-\infty, \infty)$. Functions of this type are intrinsically nonsmooth and highly inportant in the theory of variational analysis and its numerous applications, particularly to optimization and control, where they are often called the value functions (and also as the Hanilton-Jacobi-Bellman-Isaacs functions in the calculus of variations, optimal control, and differential games); see, e.g., Clarke et al. [4], Rockafellar and 
Wets [30], Vinter [34], and the references therein. Based on the refined upper estimates obtained and on subdifferential characterizations of the classical Lipschitz continuity that goes back to Rockafellar [29! in finite dimensions, we derive new efficient conditions ensuring local Lipschitz behavior for the general class of marginal functions (1.2) and their modifications in the case of infinite-dinnensional spaces; this is undoubtedly needed for many applications.

The rest of the Iilfer is organized as follows. Section 2 reviews preliminary material from variational analyis and generalized differentiation widely used to derive the main results in the subsequlit sections.

Section 3 is devotei wo coderitative calculus. The main resuit here is a new chain rule for mixed coderivative of (ompositions we label as zero chain rule. In contrast to general coderivative chain rula for hoth normal and mixed coderivatives, which inevitably require the usage of the nomini colcrivative for inner mappings in compositions, the new result applies only to mixed culerivative's of compositions at zero value $y^{*}=0$ of the coderivative argument. But this is exirtly what we need for applications to robust Lipschitzian stability!

The main topic of Siction \& is the preservation of robust Lipschitzian stability (with the corresponding calculus for the (xact bounds) under various operations on set-valued mappings. Despite its undoubted importince, this topic has not drawn much attention in the literature; some results are available in the book by Rockafellar and Wets [30] in finite dimensions and will also appear in the hook by Mordukhovich [18] in infinite-dimensional spaces. We derive refined conditions in this direction for fairly general settings based on the mentioned coderivative criteria for Lipschitzian stability and on the special zero calculus results established in Section 3. In contrast to finite dimensions, the infinite-dimensional consideration requires the usage of the recently developed calculus of sequential normal compactness, by which we mean results on preserving certain compactness-like properties in variational analysis unavoidably needed in infinite-dimensional spaces; see Section 2.

The concluding Section 5 is devoted to the study of marginal functions of type (1.2) as well as their extensions covering. in particular. the distance function to moving sets. The principal new relationship established between the singular subdifferential of (1.2) and the mixed coderivative of the generating mapping $F$ is obtained in the form

$$
\partial^{x} \mu(x) \subset \bigcup_{y=s+x}\left\{r^{*} \in X^{*}: x^{*} \in D_{Y} F(\vec{r}, \bar{y})(0)\right\}
$$

provided that $\varphi$ is locally Lipschitzian. where

$$
S(x):=\{y \in F(x) \mid M(x)=\hat{r}(x, y)\}
$$

stands for the minimum/solution map wo the parameterized optimizaticn problem in (1.2). Inclusion (1.3) improves previously known results of this type. where $D_{M}^{*}$ is replaced by the normal coderivative (1.1). Based on (1.3), we derive new conditions ensuring local Lipschitz continuity of marginal functions via the mixed coderivative of the generating mapping $F$. Taking into account the well-developed calculus for mixed coderivatives, this allows us to establish efficient results for Lipschitz continuity of marginal/value functions in specific - classes of parametric optimization problems, where $F(x)$ describe (moving) sets of feasible solutions. It covers, in particular, mathematical problems with equilibrium constraints 
(MPECs) corresponding to $F(x)$ arose as solution sets to lower level optimization problems, or-more generally-to parametric variational inequalities and complementarity relations.

Throughout the paper we use standard notation of variational analysis and generalized differentiation. Given a set-valued mapping $F: X \rightrightarrows X^{*}$ between a Banach space $X$ and its topological dual $X^{*}$, denote

$$
\begin{aligned}
\operatorname{Limsup}_{x \rightarrow \bar{x}} F(x):=\left\{x^{*} \in X^{*} \mid\right. & \exists \text { sequence } x_{k} \rightarrow \bar{x} \text { and } x_{k}^{*} \stackrel{w^{*}}{\longrightarrow} x^{*} \\
& \text { with } \left.x_{k}^{*} \in F\left(x_{k}\right) \text { for all } k \in \mathbb{N}\right\}
\end{aligned}
$$

the sequential Painleve-Kuratowski upper/outer limit with respect to the norm topology of $X$ and the weak* topulogy of $X^{*}$, where $N:=\{1,2, \ldots\}$. Recall that the norm of any homogeneous multifunction $F: X \rightrightarrows Y$ is defined by

$$
\|F\|:=\operatorname{sip}\{\|y\| y \in F(x) \text { and }\|x\| \leq 1\} \text {. }
$$

\section{Preliminaries}

This section mostly contains some preliminary material on generalized differentiation widely used in what follows. We refer the reader to the book by Mordukhovich [18] for more details and discussions. Unless otherwise stated, all the spaces under consideration are Banach. As usual, $\mathbb{B}$ and $\mathbb{B}^{*}$ stand for the closed unit balls of the space in question and its dual.

Given $\Omega \subset X$ and $\varepsilon \geq 0$, define the collection of $\varepsilon$-normals to $\Omega$ at $\bar{x} \in \Omega$ by

$$
\widehat{N}_{\varepsilon}(\bar{x} ; \Omega):=\left\{x^{*} \in X^{*} \mid \limsup _{x \stackrel{n}{a} \bar{x}} \frac{\left\langle x^{*}, x-\bar{x}\right\rangle}{\|x-\bar{x}\|} \leq \varepsilon\right\},
$$

where $x \stackrel{\Omega}{\rightarrow} \bar{x}$ means that $x \rightarrow \bar{x}$ with $x \in \Omega$. When $\varepsilon=0$, the set $\widehat{N}(\bar{x} ; \Omega):=\widehat{N}_{0}(\bar{x} ; \Omega)$ in (2.1) is a cone called the prenormal cone or the Fréchet normal cone to $\Omega$ at $\bar{x}$.

The basic/limiting normal cone $N(\bar{x}: \Omega)$ is obtained from $\widehat{N}_{\varepsilon}(x ; \Omega)$ by taking the sequential Painlevé-Kuratowski upper limit in the weak* topology of $X^{*}$ as

$$
N(\bar{x} ; \Omega):=\underset{x \in \operatorname{sins}_{\varepsilon ! 0}:}{\operatorname{Lip}} \widehat{N}_{\varepsilon}(x: \Omega),
$$

where one can put $\varepsilon=0$ when $\Omega$ is closed around $\bar{x}$ and the space $X$ is Asplund, i.e., a Banach space whose separable subspaces have separable duals. This class of spaces is sufficiently large including, in particular, every refiexive space; see, e.g., the book by Phelps [27] for more information and references.

Let $\varphi: X \rightarrow \overline{\mathbb{R}}$ be an extended-real-valued function finite at $\bar{x}$. The set

$$
\widehat{\partial}_{\varepsilon} \varphi(\bar{x}):=\left\{x^{*} \in X^{*} \mid \liminf _{x \rightarrow \bar{x}} \frac{\varphi(x)-\varphi(\bar{x})-\left\langle x^{*}, x-\bar{x}\right\rangle}{\|x-\bar{x}\|} \geq-\varepsilon\right\}
$$

is called the (Fréchet-like) $\varepsilon$-subdifferential of $\varphi$ at $\bar{x}$. The basic/limiting subdifferential of $\varphi$ at $\bar{x}$ is defined by

$$
\partial \varphi(\bar{x}):=\limsup _{\substack{x \in \underbrace{}_{\varepsilon \downarrow 0} \\ \varepsilon \downarrow j}} \widehat{\partial}_{\varepsilon} \varphi(x)
$$


where $x \stackrel{\varphi}{\rightarrow} \bar{x}$ means that $x \rightarrow \bar{x}$ and $\varphi(x) \rightarrow \varphi(\bar{x})$. Note that $\widehat{\partial}_{\varepsilon} \varphi$ can be replaced by $\widehat{\partial} \varphi:=\widehat{\partial}_{0} \varphi$ in (2.4) when $X$ is Asplund while $\varphi$ is lower semicontinuous (l.s.c.) around $\tilde{x}$. Let us mention an equivalent geometric definition of the basic subdifferential (2.4) by

$$
\partial_{\psi}(\ddot{x})=\left\{x^{*} \in X^{*} \mid\left(x^{*},-1\right) \in N((\tilde{x}, \varphi(\bar{x})) ; \operatorname{epi} \varphi)\right\}
$$

via the epigraphical $\cdots$ epi $y:=\{(x, \mu) \in X \times \mathbb{R} \mid \mu \geq \varphi(x)\}$.

The singular subdeffrential of $\hat{\sim}: X \rightarrow \overline{\mathbb{R}}$ at $\bar{x}$ is defined by

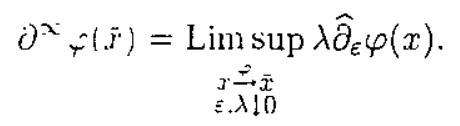

This construction make's serse only for non-Lipschitzian functions, since $\partial^{\infty} \varphi(\bar{x})=\{0\}$ if $\varphi$ is Lipschitz continuoum alumul $\bar{x}$. Vote that $\varepsilon>0$ can be omitted in (2.5) if $X$ is Asplund and $\varphi$ is l.s.c. around $i$.

Let $F: X \rightrightarrows Y$ be a set-valund mapping between Banach spaces with the graph

$$
\operatorname{gph} F:=\{(x, y) \in X \times Y \mid y \in F(x)\}
$$

The normal coderivative $D_{Y}^{*} F(\bar{x}, \bar{y}): Y^{*}=X^{*}$ of $F$ at $(\bar{x}, \bar{y})$ is defined by

$$
D_{N}^{*} F(\bar{x} \cdot \bar{y})\left(y^{*}\right):=\left\{x^{*} \subseteq X^{*} \mid\left(x^{*},-y^{*}\right) \in N((\bar{x}, \bar{y}) ; \operatorname{gph} F)\right\}
$$

and the corresponding mixed codermatue is

$$
\begin{aligned}
& D_{M}^{*} F(\vec{x}, \bar{y})\left(y^{*}\right):=\left\{x^{*}=X^{*} \Xi_{k}: 0 .\left(x_{k}, y_{k}\right) \stackrel{g p_{1} F}{\rightarrow}(\bar{x}, \bar{y}), x_{k}^{*} \stackrel{w^{*}}{\longrightarrow} x^{*},\right. \\
& \left.y_{k}^{*} \stackrel{r}{\longrightarrow} y^{*} \text { with }\left(x_{k}^{*},-y_{k}^{*}\right) \in \widehat{N}_{\varepsilon_{k}}\left(\left(x_{k}, y_{k}\right) ; \operatorname{gph} F\right)\right\} \text {, }
\end{aligned}
$$

where $\stackrel{w^{*}}{\rightarrow}$ signifies the weak* srquentiul convergence in $X^{*}$. while $\stackrel{\|\cdot\|}{\rightarrow}$ stands for the norm convergence in the dual space; we omil $\mid ;$ in whist follows. One can put $\varepsilon_{k}=0$ in (2.7) if $X$ and $Y$ are Asplund and if the graph of $F$ is r lesed around $(\bar{x}, \bar{y})$. Clearly

$$
D_{M}^{*} F(\bar{x}, \bar{y})\left(y^{*}\right) \subset D_{i}^{*} F\left(x^{*}, y\right) y^{*} \text { fur all } y^{*} \in Y^{*} \text {, }
$$

where the equality obviously holds if dim ${ }^{\prime}<x$. While the inclusion may be strict in any infinite-dimensional space $\mathcal{I}^{\prime}$ even for Lipschitz continuous and Fréchet differentiable mappings on $\mathbb{R}$. The equality

$$
\left\|D_{M}^{*} F(\bar{x}, \bar{y})\right\|_{=}=\mid D_{i}^{*} ; F(\bar{x}, \bar{y}) \|
$$

defines the class of coderivatively normal nuappings $F$ at $(\bar{x}, \bar{y})$. This class is rather large (see sufficient conditions presented in Mordukhovich [17, Proposition 3.2] and in Subsection 4.2.1 of the afore-mentioned book) particularly including all strictly differentiable mappings, convex-graph mappings, and the so-called strictly Lipschitzian mappings on Asplund spaces. The latter notion for locally Lipschitzian mappings $f: X \rightarrow Y$ into infinite-dimensional 
spaces was defined by Mordukhovich and Shao [20] via the existence of a convergent subsequence for any sequence in the form

$$
\frac{f\left(x_{k}+t_{k} v\right)-f\left(x_{k}\right)}{t_{k}} \text { as } x_{k} \rightarrow \ddot{x} \text { and } t_{k} \downarrow 0
$$

whenever $v$ belongs to some neighborhood of the origin in $X$, but it happened to be equivalent to the major version of "compactly Lipschitzian" mappings introduced and studied much earlier by Thibault [31].

One of the most fundamental differences between variational analysis in finite and infinite dimensions, crucial for many aspects of generalized differentiation and optimization, is the necessity of imposing additional compactness-like requirements in infinite-dimensional settings that ensure nontriviality while passing to the limit in the weak* topology. In this paper we use the following general properties that are automatic in finite dimensions, hold for "reasonably good" sets and mappings, and are preserved under various operations.

A set $\Omega$ is sequentially normally compact (SNC) at $\bar{x}$ if for any sequences $\varepsilon_{k} \downarrow 0, x_{k} \stackrel{\Omega}{\rightarrow} \bar{x}$, and $x_{k}^{*} \in \widehat{N}_{\varepsilon_{k}}\left(x_{k} ; \Omega\right)$ one has

$$
\left[x_{k}^{*} \stackrel{w^{*}}{\rightarrow} 0\right] \Longrightarrow\left[\left\|x_{k}^{*}\right\| \rightarrow 0\right] \text { as } k \rightarrow \infty,
$$

where $\varepsilon_{k}$ can be omitted if $X$ is Asplund and if $\Omega$ is locally closed around $\bar{x}$. The SNC condition is automatic when $\Omega$ satisfies the so-called "compactly epi-Lipschitzian" property in the sense of Borwein and Strojwas, particularly when it is convex and finite-codimensional with nonempty relative interior.

A set-valued mapping $F: X \rightrightarrows Y$ is $S N C$ at $(\bar{x}, \bar{y}) \in \operatorname{gph} F$ if its graph enjoys this property. For the case of mappings, a more subtle partial $S N C$ (i.e., PSNC) property can be defined. We say that $F$ is $P S N C$ at $(\bar{x}, \bar{y})$ if for any sequences $\varepsilon_{k} \downarrow 0,\left(x_{k}, y_{k}\right) \stackrel{\operatorname{gph} F}{\rightarrow}(\bar{x}, \bar{y})$, and $\left(x_{k}^{*}, y_{k}^{*}\right) \in \widehat{N}_{\varepsilon_{k}}\left(\left(x_{k}, y_{k}\right) ; \operatorname{gph} F\right)$ one has

$$
\left[x_{k}^{*} \stackrel{\omega^{*}}{\rightarrow} 0,\left\|y_{k}^{*}\right\| \rightarrow 0\right] \longrightarrow\left[\left\|x_{k}^{*}\right\| \rightarrow 0\right] \text { as } k \rightarrow \infty,
$$

where $\varepsilon_{k}=0$ in the Asplund space and closed graph setting. The PSNC property always holds when $F$ is Lipschitz-like around $(\bar{x}, \bar{y})$ in the following sense of Aubin [1]: there exist neighborhoods $U$ of $\vec{x}$ and $V$ of $\bar{y}$ as well as modulus $(\geq 0$ such that

$$
F(u) \cap V \subset F(v)+\ell\|u-v\| \mathbb{B} \text { whenever } u, v \in U .
$$

This reduces to the classical (Hausdorff) local Lipschitzian behavior of $F$ around $\bar{z}$ corresponding to $V=Y$ in (2.8). The infimum of all Lipschitzian moduli $\ell$ in (2.8) is called the exact Lipschitzian bound of $F$ around $(\tilde{x}, \bar{y})$ and is denoted by $\operatorname{lip} F(\bar{x}, \bar{y})$.

The Lipschitz-like property of $F$ around $(\bar{x}, \bar{y})$ is known to be equivalent to the metric regularity and covering/linear openness properties of the inverse mapping $F^{-1}$ around $(\bar{y}, \bar{x})$, with the exact bound relationships

$$
\operatorname{reg} F^{-1}(\bar{y}, \ddot{x})=\operatorname{cov} F^{-1}(\bar{y}, \bar{x})^{-1}=\operatorname{lip} F(\bar{x}, \bar{y}) .
$$

These three equivalent properties play a fundamental role in many aspects of nonlinear analysis, especially those related to optimization. 
An extended-real-valued functions $\varphi$ on $X$ is sequentially normally epi-compact (SNEC) at $\bar{x}$ if its epigraph is SNC at $(\bar{x}, \varphi(\bar{x}))$. This property always holds for locally Lipschitzian functions and their appropriate extensions.

Finally in this section, we formulate two fundamental results of variational analysis that give coderivative/subdifferential characterizations of robust Lipschitzian properties for setvalued mappings and extended-real-valued functions, respectively. These results form the basis for developments in this paper.

Theorem 2.1 (coderivative/subdifferential characterizations of local Lipschitz continuity). Let the spaces under consideration are Asplund. Then the following hold:

(i) A closed-graph mapping $F: X \rightrightarrows Y$ is Lipschitz-like around $(\bar{x}, \bar{y}) \in \operatorname{gph} F$ if and only if $D_{M}^{*} F(\bar{x}, \bar{y})(0)=\{0\}$ and $F$ is PSNC at $(\bar{x}, \bar{y})$. In this case

$$
\left\|D_{i}^{*} F(\bar{x}, \bar{y}) \mid \leq \operatorname{lip} F(\bar{x}, \bar{y}) \leq\right\| D_{N}^{*} F(\bar{x}, \bar{y}) \|
$$

for the exact Lipschitzian bound of $F$ around $(\bar{x}, \bar{y})$, where the upper estimate is fulfilled when $\operatorname{dim} X<\infty$. Thus

$$
\operatorname{lip} F(\bar{x}, \bar{y})=\left\|D_{i}^{*} F(\bar{x}, \bar{y})\right\|=\left\|D_{N}^{*} F(\bar{x}, \bar{y})\right\|
$$

if in addition $F$ is coderivatively normal at $(\bar{x}, \bar{y})$.

(ii) A l.s.c. function $\varphi: X \rightarrow \bar{R}$ finite at $\bar{x}$ is locally Lipschitzian around $\bar{x}$ if and only if $\partial^{\infty} \varphi(\bar{x})=\{0\}$ and $f$ is $S N E C$ at this point.

\section{Coderivative Calculus}

In this section we derive a new zero chain rule for mixed coderivatives and present some of its useful subsequences. Let us start with the formulation of a fuzzy sum rule for $\varepsilon$-coderivai is of set-valued mappings $F: X \Rightarrow Y$ at $(\bar{x}, \bar{y}) \in$ gph $F$ defined by

$$
\widehat{D}_{\varepsilon}^{*} F(\bar{x} \cdot \bar{y})\left(y^{*}\right):=\left\{x^{*} \in X^{*} \mid\left(x^{*},-y^{*}\right) \in \widehat{N}_{\varepsilon}((\bar{x}, \bar{y}) ; \operatorname{gph} F)\right\}
$$

via the set of $\varepsilon$-normals given in (2.1). The following result. used in the proof of the main theorem in this section, is a simplification of a morr ge'ne'wal fuzzy rule for $\varepsilon$-coderivatives established by Mordukhorich and Shao 22!

Lemma 3.1 (fuzzy sum rule for $\Xi$-coderivatives). Let $X$ and $Y$ be Asplund spaces, let $F_{i}: X \rightrightarrows Y, i=1,2$, be set-valued mappings. and let $\leq \geq 0$. Fix

$$
\bar{y} \in\left(F_{1}+F_{2}\right)(\bar{x}) \text { with } \bar{y}=\bar{y}_{1}+\bar{y}_{2} \text { and } \bar{y}_{t} \in F_{l}(\bar{x}) \text { as } i=1,2
$$

and assume that either $F_{1}$ is Lipschitz-like around $\left(\bar{x}_{1} \bar{y}_{1}\right)$. or $F_{2}$ is Lipschitz-like around $\left(\bar{x}, \bar{y}_{2}\right)$. Then for every $x^{*} \in \widehat{D}_{\varepsilon}^{*}\left(F_{1}+F_{2}\right)(\bar{x}, \bar{y})\left(y^{*}\right)$ and every $\eta>0$ there are

$$
\left(x_{i}, y_{i}\right) \in \operatorname{gph} F_{i} \cap\left[\left(\bar{x}, \bar{y}_{i}\right)+\eta \mathbb{B}\right] \text { and } x_{i}^{*} \in \widehat{D}^{*} F_{i}\left(x_{i}, y_{i}\right)\left(y_{i}^{*}\right), \quad i=1,2,
$$

satisfying the estimates

$$
\left\|y_{i}^{*}-y^{*}\right\| \leq \varepsilon+\eta \text { and }\left\|x^{*}-x_{1}^{*}-x_{2}^{*}\right\| \leq \varepsilon+\eta .
$$


Recall that a set-ralued mapping $S: X \rightrightarrows Y$ is inner semicontinuous at $(\bar{x}, \bar{y}) \in \operatorname{gph} S$ if for every sequence $x_{k} \rightarrow \bar{x}$ with $S\left(x_{k}\right) \neq \emptyset$ there is a subsequence of $y_{k} \in S\left(x_{k}\right)$ converging to $\bar{y}$ as $k \rightarrow \infty$. The mapping $S$ is inner semicompact at $\bar{x}$ with $S(\bar{x}) \neq \emptyset$ if for every sequence $x_{k} \rightarrow \bar{x}$ with $S\left(x_{k}\right) \neq \emptyset$ there is a sequence $y_{k} \in S\left(x_{k}\right)$ containing a convergent subsequence.

Now given two ser-valued mappings $G: X \rightrightarrows Y$ and $F: X \times Y \rightrightarrows Z$, consider their general composition werined by

$$
(F \circ G)(x):=\bigcup_{y \in G(x)} F(x, y)
$$

Theorem 3.2 (zero coderivative chain rule for general compositions of set-valued mappings). Fix $\bar{z} \in: F \cdot G(\bar{F})$ and assume that the mappings $F$ and $G$ are closed-graph around the reference mimts and that the spaces $X, Y$, and $Z$ are Asplund. Define

$$
S(r . z):=\{y \in G(x) \mid z \in F(x, y)\} .
$$

(i) Suppose that $S$ in mur scmumtinuous at $((\bar{x}, \bar{z}), \bar{y}) \in \operatorname{gph} S$ and that $F$ is Lipschitzlike around $((\bar{x}, \vec{y}), \bar{z})$. Then

$$
D_{M}^{*}(F \circ G)(\bar{x}, \bar{z})(0) \subset\left\{x^{*} \in X^{*} \mid x^{*} \in D_{M}^{*} G(\bar{x}, \bar{y})(0)\right\} .
$$

(ii) Suppose that $S$ is inner semucompact at $(\bar{x}, \bar{z})$ and that $F$ is Lipschitz-like around $((\bar{x}, \bar{y}), \bar{z})$ for all $\bar{y} \in S(\bar{x}, \bar{z})$. Then

$$
D_{M}^{*}(F \circ G)(\tilde{x}, \bar{z})(0) \subset \bigcup_{y \in S(r, z)}\left\{x^{*} \in X^{*} \mid x^{*} \in D_{M}^{*} G(\bar{x}, \bar{y})(0)\right\} .
$$

Proof. It is sufficient to justify assertion (i). since the proof of (ii) is similar. Define the auxiliary mapping

$$
H(x, y):=F(x, y)+\Delta((x, y): \operatorname{gph} G),
$$

where the set indication mapping $\Delta(: \Omega \Omega)$ is given by $\Delta(u: \Omega):=0$ if $w \in \Omega$ and $\Delta(w ; \Omega):=\emptyset$ otherwise. Fix an arbitrary element $r^{*} \in D_{y}^{*}(F \circ(\xi)(\bar{r}, z)(0)$ and, using definition $(2.7)$ of the mixed coderivative. find $\Sigma_{k} .0 . x_{k} \rightarrow \bar{x} . z_{k} \rightarrow \Sigma_{k} x_{k}^{*} x^{*}$, and $z_{k}^{*} \rightarrow 0$ (converging by norm) as $k \rightarrow \infty$ such that

$$
z_{k} \in(F \circ G)\left(x_{k}\right) \text { and }\left(x_{k}^{*}-z_{k}^{*}\right) \in \hat{\Gamma}_{s_{k}}\left(\left(x_{k} \circ z_{k}\right): \operatorname{gph}(F \circ G)\right), k \in \mathbb{N} .
$$

Since $S$ is inner semicontinuous at $((\bar{x}, \bar{z}), \bar{y})$. find $y_{k} \in S\left(x_{k}, z_{k}\right)$ whose subsequence converges (without relabeling) to some $\bar{y}$. We have $\bar{y} \in S(\bar{r}$. $z$ ) by the closed-graph assumptions of the theorem. It is easy to observe from (3.2) that

$$
\left(x_{k}^{*}, 0,-z_{k}^{*}\right) \in \widehat{N}_{\varepsilon_{k}}\left(\left(x_{k}, y_{k}, z_{k}\right): \operatorname{gph} H\right) \Longleftrightarrow\left(x_{k}^{*} .0\right) \in \widehat{D}_{\varepsilon_{k}}^{*} H\left(x_{k}, y_{k}, z_{k}\right)\left(z_{k}^{*}\right), \quad k \in \mathbb{N},
$$

by the construction of $\varepsilon$-coderivatives. Taking into account the sum structure of the mapping $H$ in (3.1) and using Lemma 3.1 along a fixed sequence $\eta_{k} \downarrow 0$, find

- $\left(\left(x_{1 k}, y_{1 k}\right), z_{1 k}\right) \in \operatorname{gph} F, \quad\left(x_{2 k}, y_{2 k}\right) \in \operatorname{gph} G, \quad\left(x_{1 k}^{*}, y_{1 k}^{*}\right) \in \widehat{D}^{*} F\left(x_{1 k}, y_{1 k}, z_{1 k}\right)\left(z_{1 k}^{*}\right)$, and $\left(x_{2 k}^{*}, y_{2 k}^{*}\right) \in \widehat{N}\left(\left(x_{2 k}, y_{2 k}\right) ; g p h G\right)$ as $k \in \mathbb{N}$ 
satisfying the estimates

$$
\begin{aligned}
& \left\|\left(x_{1 k}, y_{1 k}, z_{1 k}\right)-\left(x_{k}, y_{k}, z_{k}\right)\right\| \leq \eta_{k}, \quad\left\|\left(x_{2 k}, y_{2 k}\right)-\left(x_{k}, y_{k}\right)\right\| \leq \eta_{k}, \\
& \left\|\left(x_{k}^{*}, 0\right)-\left(x_{1 k}^{*}, y_{1 k}^{*}\right)-\left(x_{2 k}^{*}, y_{2 k}^{*}\right)\right\| \leq \varepsilon_{k}+\eta_{k}, \quad\left\|z_{1 k}^{*}-z_{k}^{*}\right\| \leq \varepsilon_{k}+\eta_{k} .
\end{aligned}
$$

Since $\left\|z_{k}^{*}\right\| \rightarrow 0$ and $\left\|z_{1 k}^{*}-z_{k}^{*}\right\| \leq \varepsilon_{k}+\eta_{k}$, we have $\left\|z_{1 k}^{*}\right\| \rightarrow 0$ as $k \rightarrow \infty$. The Lipschitz-like assumption on $F$ ensures, by Theorem $2.1(\mathrm{i})$, that $F$ is PSNC at $((\bar{x}, \bar{y}), \bar{z})$, which implies that $\left\|\left(x_{1 k}^{*}, y_{1 k}^{*}\right)\right\| \rightarrow 0$. Combining this with

$$
\left\|x_{k}^{*}-x_{1 k}^{*}-x_{2 k}^{*}\right\| \leq \varepsilon_{k}+\eta_{k}, \quad\left\|y_{1 k}^{*}+y_{2 k}^{*}\right\| \leq \varepsilon_{k}+\eta_{k}, \quad \text { and } x_{k}^{*} \stackrel{w^{*}}{\longrightarrow} x^{*},
$$

we conclude that $x_{2 k}^{*} \stackrel{u^{*}}{\rightarrow} 0$ and $\left\|y_{2 k}^{*}\right\| \rightarrow 0$ as $k \rightarrow \infty$, and thus $x^{*} \in D_{M}^{*} G(\bar{x}, \bar{y})(0)$. This completes the proof of the theorem.

Theorem 3.2 immediately applies, without any change, to standard compositions of set-valued mappings when $F=F(y)$. and also to special cases of single-valued mappings in compositions. Let us present its corollary for the case of single-valued inner mappings $G=g: X \rightarrow Y$ when the inner semicontinuity assumption in (i) holds automatically.

Corollary 3.3 (zero chain rule with single-valued inner mappings). Under the general assumptions of Theorem 3.2, suppose that $g: X \rightarrow Y$ is continuous around $\bar{x}$ while $F: Y \rightrightarrows Z$ is Lipschitz-like around $(g(\bar{x}), \bar{z})$. Then

$$
D_{M}^{*}(F \circ g)(\bar{x}, \bar{z})(0) \subset\left\{x^{*} \in X^{*} \mid x^{*} \in D_{M}^{*} g(\bar{x})(0)\right\} .
$$

Another corollary of Theorem 3.2 concerns the so-called reversed mixed coderivative of $F: X \rightrightarrows Y$ at $(\bar{x}, \bar{y}) \in \operatorname{gph} F$ defined by

$$
\widetilde{D}_{M}^{*} F(\bar{x}, \bar{y})\left(y^{*}\right):=\left\{x^{*} \in X^{*} \mid y^{*} \in-D_{M}^{*} F^{-1}(\bar{y}, \bar{x})\left(-x^{*}\right)\right\},
$$

which is convenient for characterizing the metric regularity and covering/openness properties; cf. Mordukhovich [16], Mordukhovich and Shao [23], and Penot [26]. The result of Theorem 3.2 allows us to derive a useful relationship for the kernel

$$
\operatorname{ker} \widetilde{D}_{M}^{*} F(\bar{x}, y):=\left\{y^{*} \in Y^{*} \mid 0 \in \tilde{D}_{M}^{*} F(\bar{x}, \bar{y})\left(y^{*}\right\}\right.
$$

of the reversed mixed coderivative under compositions.

Corollary 3.4 (kernels of reversed mixed coderivatives under compositions). Let $\bar{z} \in(F \circ G)(\bar{x})$, where $G: X \rightrightarrows Y$ and $F: Y \rightrightarrows Z$ are closed-graph mappings between Asplund spaces. Assume that the mapping $S(x, z):=G(x) \cap F^{-1}(z)$ is inner semicompact. at $(\bar{x}, \bar{z})$ and that $G$ is metrically regular around $(\bar{x}, \bar{y})$ for all $\bar{y} \in S(\bar{x}, \bar{z})$. Then

$$
\operatorname{ker} \widetilde{D}_{M}^{*}(F \circ G)(\bar{x}, \bar{z}) \subset \bigcup_{\bar{y} \in S(\bar{x}, \bar{z})} \operatorname{ker} \widetilde{D}_{M}^{*} F(\bar{y}, \bar{z})
$$


Proof. Fix $z^{*} \in \operatorname{ker} \widetilde{D}_{M}^{*}(F \circ G)(\bar{x}, \bar{z})$ and use the identity

$$
(F \circ G)^{-1}=G^{-1} \circ F^{-1} .
$$

Then the relation $0 \in \tilde{D}_{M}^{*}(F \circ G)(\bar{x}, \bar{z})\left(z^{*}\right)$ is equivalent to $-z^{*} \in D_{M}^{*}(F \circ G)^{-1}(\bar{z}, \bar{x})(0)$. Since the metric regularity of $G$ around $(\bar{x}, \bar{y})$ is equivalent to the Lipschitz-like property of $G^{-1}$ around $(\bar{y}, \bar{x})$, we get from Theorem 3.2 that

$$
\text { - } z^{*} \in D_{M}^{*} F^{-1}(\bar{z}, \bar{y})(0) \text { for some } \bar{y} \in S(\bar{x}, \bar{z})
$$

This gives $0 \in \widetilde{D}_{M}^{*} F \quad(H, \bar{F})\left(z^{*}\right)$, and thus $z^{*} \in \operatorname{ker} \widetilde{D}_{M}^{*} F(\bar{y}, \bar{z})$, which completes the proof of the corollary.

\section{Robust Lipschitzian Stability}

This section concerms calculus of Lipschitzian stability by which we understand efficient conditions ensuring the preservation of robust Lipschitzian properties under various operations. As usual, we pay the main attention to the general Lipschitz-like property of set-valued mappings. The calculus/preservation results established for this property easily imply the corresponding results for the classical local Lipschitzian property, as well as for metric regularity and covering via inverse mappings. Observe that we also obtain relationships for the corresponding catact bounds under various compositions.

Our first result in this section 'nsures the preservations of the Lipschitz-like property under general compositions of sct-vialued mappings.

Theorem 4.1 (Lipschitz-like property under general compositions). Considering closed-graph mappings $F: X \times Y^{\circ} \rightrightarrows Z$ and $G: X=Y^{r}$ between Asplund spaces, take $a$ point $\bar{z} \in(F \circ G)(\bar{x})$ such that gph $(F \circ G)$ is locally closed around $(\bar{x}, \bar{z})$ and define

$$
S(x . z):=\{u \in C(x)\}=\in F(x, y)\} .
$$

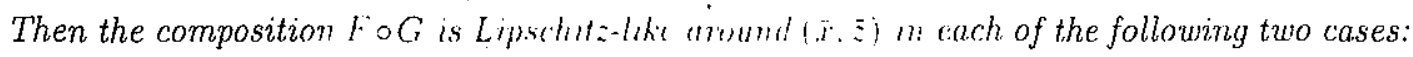

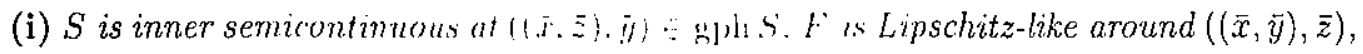
and $G$ is Lipschitz-like around $(\bar{i}, \bar{i})$.

(ii) $S$ is inner semicompact at $(\bar{x}, \bar{\xi})$, and for rery $\bar{y} \in S(\bar{x}, \bar{z})$ the mapping $F$ is Lipschitz-like around $((\bar{x}, \bar{y}) . \bar{z})$ while $G$ is Lipschitz-lite around $(\bar{x}, \bar{y})$.

Proof. It is sufficient to justify (i) observing that the proof of (ii) is similar. Since $G$ is Lipschitz-like around $(\bar{x}, \bar{y})$. we get from Theorem 2.1(i) that $D_{M}^{*} G(\bar{x}, \bar{y})(0)=\{0\}$. The new zero chain rule from Theorem 3.2 allows us to conclude that $D_{M}^{*}(F \circ G)(\bar{x}, \bar{z})(0)=\{0\}$. Thus, by the characterization of Theorem 2.I(i), it remains to check that the composition $F \circ G$ is $P S N C$ at $(\bar{x}, \bar{z})$.

To proceed, we take any sequences $\left(x_{k}, z_{k}, x_{k}^{*}, z_{k}^{*}\right)$ such that $\left(x_{k}, z_{k}\right) \rightarrow(\bar{x}, \bar{z}), x_{k}^{*} \stackrel{w^{*}}{\rightarrow} 0$, $\left\|z_{k}^{*}\right\| \rightarrow 0$ as $k \rightarrow \infty, z_{k} \in(F \circ G)\left(x_{k}\right)$. and

$$
\left(x_{k}^{*},-z_{k}^{*}\right) \in \hat{N}\left(\left(x_{k}, z_{k}\right) ; \operatorname{gph}(F \circ G)\right) \text { for all } k \in L
$$


observe that we omitted $\varepsilon_{k} \downarrow 0$ in comparison with the general definition of the PSNC property, since the spaces $X \times Z$ are Asplund and the graph of $F \circ G$ is locally closed. By the inner semicontinuous assumption on $S$ at $((\bar{x}, \bar{z}), \bar{y})$, we get $y_{k} \in S\left(x_{k}, z_{k}\right)$ such that $y_{k} \rightarrow \bar{y}$ along a subsequence still labeled $\left\{y_{k}\right\}$. Then arguing similarly to the proof of Theorem 3.2 with the usage the coderivative fuzzy sum rule from Lemma 3.1 , fix $\eta_{k} \downarrow 0$ and find by (4.1) some sequences

$$
\begin{aligned}
& \left(x_{1 k}, y_{1 k}, z_{1 k}\right) \stackrel{\operatorname{sph} F}{\longrightarrow}(\bar{x}, \bar{y}, \bar{z}), \quad\left(x_{2 k}, y_{2 k}\right) \stackrel{\operatorname{gph} G}{\longrightarrow}(\bar{x}, \bar{y}), \\
& \left(x_{1 k}^{*}, y_{1 k}^{*}\right) \in \hat{D}^{*} F\left(x_{1 k}, y_{1 k}, z_{1 k}\right)\left(z_{1 k}^{*}\right), \quad \text { and }\left(x_{2 k}^{*}, y_{2 k}^{*}\right) \in \widehat{N}\left(\left(x_{2 k}, y_{2 k}\right) ; \operatorname{gph} G\right)
\end{aligned}
$$

satisfying the estimates

$$
\left\|\left(x_{k}^{*}, 0\right)-\left(x_{1 k}^{*}, y_{1 k}^{*}\right)-\left(x_{2 k}^{*}, y_{2 k}^{*}\right)\right\| \leq \eta_{k} \text { and }\left\|z_{1 k}^{*}-z_{k}^{*}\right\| \leq \eta_{k} .
$$

It follows from (4.3) that $\left\|z_{1 k}^{*}\right\| \rightarrow 0$. By the PSNC property of $F$ implied by its assumed Lipschitz-like property; we get from (4.2) that $\left\|\left(x_{1 k}^{*}, y_{1 k}^{*}\right)\right\| \rightarrow 0$. Then (4.3) yields that $\left\|y_{2 k}^{*}\right\| \rightarrow 0$, and hence the Lipschitz-like property of $G$ ensures by (4.2) that $\left\|x_{2 k}^{*}\right\| \rightarrow 0$. This finally gives by (4.3) that $\left\|x_{k}^{*}\right\| \rightarrow 0$, which justifies the PSNC property of $F \circ G$ and completes the proof of the theorem.

Theorem 4.1 automatically applies to the standard compositions of set-valued mappings with $F=F(y)$. The next result gives more: it establishes a calculus rule for the exact Lipschitzian bounds under some additional assumptions.

Theorem 4.2 (Lipschitzian bounds under compositions). Let $G: X \rightrightarrows Y$ and $F: Y \rightrightarrows Z$ be closed-graph mappings between Asplund spaces. Fix $\bar{z} \in(F \circ G)(\bar{x})$ such that $\operatorname{gph}(F \circ G)$ is locally closed around $(\bar{x}, \bar{z})$ and form the mapping $S(x, z)$ as in Theorem 4.1. The following assertions hold:

(i) Assume that $S$ is inner semicontinuous at $((\bar{x}, \bar{z}), \bar{y}) \in \operatorname{gph} S$. Then the composition $F \circ G$ is Lipschitz-like around $(\bar{x}, \bar{z})$ provided that $G$ is Lipschitz-like around $(\bar{x}, \bar{y})$ and that $F$ is Lipschitz-like around $(\bar{y}, \bar{z})$. If in addition $\operatorname{dim} X<\infty$ and the mappings $G$ and $F$ are coderivatively normal at $(\bar{x}, \bar{y})$ and $(\bar{y}, \bar{z})$, respectively, then

$$
\operatorname{lip}(F \circ G)(\bar{x}, \bar{z}) \leq \operatorname{lip} G(\bar{x}, \bar{y}) \cdot \operatorname{lip} F(\bar{y}, \bar{z}) .
$$

(ii) Assume that $S$ is inner semicompact at $(\bar{x}, \bar{z})$. Then $F \circ G$ is Lipschitz-like around $(\bar{x}, \bar{z})$ provided that $G$ is Lipschitz-like around $(\bar{x}, \bar{y})$ and $F$ is Lipschitz-like around $(\bar{y}, \bar{z})$ for all $\bar{y} \in S(\bar{x}, \bar{z})$. Moreover, one has

$$
\operatorname{lip}(F \circ G)(\bar{x}, \bar{z}) \leq \max _{\bar{y} \in S(\bar{x}, \bar{z})} \operatorname{lip} G(\bar{x}, \bar{y}) \cdot \operatorname{lip} F(\bar{y}, \bar{z})
$$

if in addition $\operatorname{dim} X<\infty$ while both $G$ and $F$ are coderivatively normal at $(\bar{x}, \bar{y})$ and $(\bar{y}, \bar{z})$, respectively, for all $\bar{y} \in S(\bar{x}, \bar{z})$.

Proof. First we justify the exact bound formula (4.4) in assertion (i). It follows from Theorem 4.5 in Mordukhovich [16] that the chain rule

$$
D^{*}(F \circ G)(\bar{x}, \vec{z})\left(z^{*}\right) \subset D_{N}^{*} G(\vec{x}, \bar{y}) \circ D^{*} F(\bar{y}, \bar{z})\left(z^{*}\right), \quad z^{*} \in Z^{*},
$$


holds for both normal $\left(D^{*}=D_{N}^{*}\right)$ and mixed $\left(D^{*}=D_{M}^{*}\right)$ coderivatives of Lipschitzlike mappings between Asplund spaces; observe that the normal coderivative of the inner mapping $G$ is used in (4.6) in both cases of $D^{*}=D_{N}^{*}, D_{M}^{*}$. Taking into account the well-known relationship

$$
\left\|H_{1} \circ H_{2}\right\| \leq\left\|H_{1}\right\| \cdot\left\|H_{2}\right\|
$$

for the norms of any positively homogeneous set-valued mappings, we derive (4.4) from the chain rule (4.6) applied to both coderivatives $D^{*}=D_{N}^{*}, D_{M}^{*}$ and from the bound estimates of Theorem 2.1(i) held under the assumptions made.

The proof of (4.j) in assertion (ii) is similar. We need to observe only that the "max" in (4.5) is achieved, since the set $S(\bar{r}, \bar{z})$ is compact in the setting under consideration and the real-valued function $\operatorname{lip} H(\cdot)$ is upper semicontinuous on the graph of any Lipschitz-like set-valued mapping $H(\cdot)$.

It is worth mentioning that the local closed-graph assumptions of Theorems 4.1 and 4.2 automatically hold when both $F$ and $G$ are locally closed-graph and the mappings $S$ therein are inner semicontinuous/semicompact around the reference points.

The following consequence of Theorem 4.2 specifies its assumptions for the case of singlevalued inner mappings.

Corollary 4.3 (Lipschitzian properties of compositions with single-valued inner mappings). Let $\bar{z} \in\left(F \circ G^{\prime}\right)(\bar{x})$. wher $F: J^{\prime} \rightrightarrows Z$ is locally closed-graph around $(g(\bar{x}), \bar{z})$ while $g: X \rightarrow Y$ is locally Lipschtzian around $\bar{x}$ in the Asplund space setting. Then $F \circ g$ is Lipschitz-like around $(\bar{x}, \bar{z})$ provided that $F$ is Lipschitz-like around $(g(\bar{x}), \bar{z})$. Moreover,

$$
\operatorname{lip}(F \circ g)(\bar{x} . \bar{z}) \leq \operatorname{lip} g(\bar{x}) \cdot \operatorname{lip} F(g(\bar{x}) . \bar{z})
$$

if in addition $X$ is finite-dimensional. $g$ is strictly Lipschitzian at $\bar{x}$, and $F$ is coderivatively normal at $(g(\bar{x}), \bar{z})$.

Proof. To derive this result from Theorem 4.2. it remains to observe that any strictly Lipschitzian mapping $g: X \rightarrow Y^{*}$ with an Asplund domin space $X$ is coderivatively normal at the reference point: see Mordukbovich $\{18\}$.

The composition results obtained above have many specifications corresponding to particular choices of mappings $F$ and $G$. Next we present conditions ensuring the preservation of Lipschitzian stability under summation of set-valued mappings. For brevity, consider only the "inner semicompact" case.

Theorem 4.4 (Lipschitz-like property under summation). Let $F_{i}: X \rightrightarrows Y, i=$ 1,2, be closed-graph mappings between Asplund spaces. Take $\bar{y} \in\left(F_{1}+F_{2}\right)(\ddot{x})$ such that $\operatorname{gph}\left(F_{1}+F_{2}\right)$ is locally closed around $(\bar{x}, \vec{y})$ and consider the mapping $S: X \times Y \rightrightarrows Y^{2}$ defined by

$$
S(x, y):=\left\{\left(y_{1}, y_{2}\right) \in Y^{2} \mid y_{1} \in F_{1}(x), y_{2} \in F_{2}(x), y_{1}+y_{2}=y\right\}
$$


Assume that $S$ is inner semicompact at $(\bar{x}, \bar{y})$. Then $F_{1}+F_{2}$ is Lipschitz-like around $(\bar{x}, \bar{y})$ provided that $F_{1}$ is Lipschitz-like around $\left(\bar{x}, \bar{y}_{1}\right)$ and $F_{2}$ is Lipschitz-like around $\left(\bar{x}, \bar{y}_{2}\right)$ for every $\left(\bar{y}_{1}, \bar{y}_{2}\right) \in S(\bar{x}, \bar{y})$. Moreover,

$$
\operatorname{lip}\left(F_{1} \cdots F_{2}\right)(\bar{x}, \bar{y}) \leq \max _{\left(\bar{y}_{1}, \bar{y}_{2}\right) \in S(\bar{x}, \bar{y})}\left\{\operatorname{lip} F_{1}\left(\bar{x}, \bar{y}_{1}\right)+\operatorname{lip} F_{1}\left(\bar{x}, \bar{y}_{1}\right)\right\}
$$

if $X$ is finite-dimensunal and ench mapping $F_{i}$ is coderivatively normal at $\left(\bar{x}, \bar{y}_{i}\right), i=1,2$, for all $\left(\bar{y}_{1}, \bar{y}_{2}\right) \in S(\bar{x},:$.

Proof. It follows frun. Murdukhorich [16, Theorem 4.2] that the sum rule

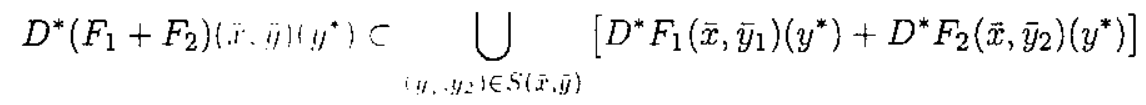

holds for both coderivitiven $L^{*}=D_{X} . D_{Y}^{*}$ under the assumptions made. Putting $y^{*}=0$ in (4.8) with $D^{*}=D_{\text {ir }}$. we ger herem 2.1 (i) that

$$
D_{i /}\left(F_{1}+F_{2}\right)(\bar{x}, \bar{y})(0)=\{0\} \text {. }
$$

Furthermore, the PSNC property of the sum $F_{1}+F_{2}$ follows from the PSNC calculus result by Mordukhovich and Wang [24. Theorem 5.1]. Thus, by the converse implication of Theorem 2.1(i), we conclude thint $F_{1}+F_{2}$ is Lipschitz-like at $(\bar{x}, \bar{y})$. Finally, using the chain rule (4.8) for both coderivatives $D^{*}=D_{N}^{*} \cdot D_{M}^{*}$ and the obvious inequality

$$
\mid H_{1}+H_{2}\|\leq\| H_{1}\|+\| H_{2} \|
$$

for the norms of positive homogencous nultifunctions. we arrive at the exact bound formula (4.7) similarly to the proof of Theorem 4.2 .

Next, give set-valued mappings $F_{1}: X \Rightarrow Y$ for $i=1,2$ and $h: Y_{1} \times Y_{2} \rightarrow Z$, consider the so-called $h$-composition

$$
\left(F_{1} \diamond F_{2}\right)(x):=\bigcup\left\{h\left(y_{1}, y_{2}\right) \mid y_{1} \in F_{1}(x) . y_{2} \in F_{2}(x)\right\}
$$

that covers various binary operatoms: see. Mordukhorich :15] and Mordukhovich and Shao [21]. The following result ensuring the preservationi of Lipsclsitz stability under $h$-compositions, is a consequence of Theorems 4.2 and 4.4 .

Corollary 4.5 (Lipschitz-like property under $/ 1$-compositions). Let $F_{i}: X \rightrightarrows Y_{i}$ for $i=1,2$, and let $h: Y_{1} \times Y_{2} \rightarrow Z$ be mappmgs betucen Asplund spaces. Define $S: X \times$ $Z \Rightarrow Y_{1} \times Y_{2}$ by

$$
S(x, z):=\left\{\left(y_{1}, y_{2}\right) \in Y_{1}^{\prime} \times Y_{2} \mid y_{2} \in F_{1}(x), z=h\left(y_{1}, y_{2}\right)\right\}
$$

and, given $\bar{z} \in\left(F_{1} \stackrel{h}{\diamond} F_{2}\right)(\bar{x})$, suppose that $S$ inner semicompact at $(\bar{x}, \bar{z})$. Assume also that for every $\bar{y}=\left(\bar{y}_{1}, \bar{y}_{2}\right) \in S(\bar{x}, \bar{z})$ the mappings $F_{2}$ are closed-graph and Lipschitz-like around $\left(\bar{x}, \bar{y}_{1}\right)$ and $\left(\bar{x}, \bar{y}_{2}\right)$, respectively, and that the operation $h$ is locally Lipschitzian around $\left(\bar{y}_{1}, \bar{y}_{2}\right)$. Then $F_{1} \stackrel{h}{\diamond} F_{2}$ is Lipschitz-like around $(\bar{x}, \bar{z})$. 
Proof. Define $F: X \Rightarrow Y_{1} \times Y_{2}$ by $F(x):=\left(F_{1}(x), F_{2}(x)\right)$ and observe that $F=\tilde{F}_{1}+\tilde{F}_{2}$, where $\tilde{F}_{1}(x):=\left(F_{1}(x), 0\right)$ and $\tilde{F}_{2}(x):=\left(0, F_{2}(x)\right)$. It follows from Theorem 4.4 that $F$ is is Lipschitz-like around $(\bar{x}, \bar{y})$ for all $\vec{y}=\left(\bar{y}_{1}, \bar{y}_{2}\right) \in S(\bar{x}, \bar{z})$. Since clearly

$$
\left(F_{1} \stackrel{h}{\diamond} F_{2}\right)(x)=(h \circ F)(x)
$$

and since $h$ is locally Lipschitzian, we apply now Theorem 4.2 to the latter composition and thus complete the proof.

Finally, let us derive a useful corollary of Theorem 4.2 that ensures the preservation of metric regularity and covering properties under compositions with calculus of the corresponding exact bounds.

Corollary 4.6 (metric regularity and covering properties under compositions). Let $\bar{z} \in(F \circ G)(\bar{x})$, where $G: X \rightrightarrows Y$ and $F: Y \rightrightarrows Z$ are closed-graph mappings between Asplund spaces. Fix $\bar{z} \in(F \circ G)(\bar{x})$ such that $\operatorname{gph}(F \circ G)$ is locally closed around $(\bar{x}, \bar{z})$. The following assertions hold:

(i) Assume that the set-valued mapping $S(x, z)$ from Theorem 4.2 is inner semicontinuous at $((\bar{x}, \bar{z}), \bar{y})$. Then $F \circ G$ is metrically regular (has the covering property) around $(\bar{x}, \bar{z})$ provided that $G$ is metrically regular (has the covering property) around $(\bar{x}, \vec{y})$ and that $F$ is metrically regular (has the covering property) around $(\bar{y}, \bar{z})$. If in addition $\operatorname{dim} Z<\infty$ and both $F^{-1}$ and $G^{-1}$ are coderivatively normal at $(\bar{z}, \bar{y})$ and $(\bar{y}, \bar{x})$, respectively, then

$$
\begin{aligned}
& \operatorname{reg}(F \circ G)(\bar{x} \cdot \bar{z}) \leq \operatorname{reg} G(\bar{x}, \bar{y}) \cdot \operatorname{reg} F(\bar{y}, \bar{z}), \\
& \operatorname{cov}(F \circ G)(\bar{x} \cdot \bar{z}) \geq \operatorname{cov} G(\bar{x}, \bar{y}) \cdot \operatorname{cov} F(\bar{y}, \bar{z}) .
\end{aligned}
$$

(ii) Assume that $S(x, z)$ is inner semicompact at $(\bar{x}, \bar{z})$. Then $F \circ G$ is metrically regular (has the covering property) around $(\bar{x}, \bar{z})$ provided that $G$ is metrically regular (has the covering property) around $(\bar{x}, \bar{y})$ and that $F$ is metrically regular (has the covering property) around $(\bar{y}, \bar{z})$ for every $\bar{y} \in S(\bar{x}, \bar{z})$. If in addition $\operatorname{dim} Z<\infty$ and for every $\bar{y} \in S(\bar{x}, \bar{z})$ both $F^{-1}$ and $G^{-1}$ are coderivatively normal at $(\bar{z}, \bar{y})$ and $(\bar{y}, \bar{x})$, respectively, then

$$
\begin{aligned}
& \operatorname{reg}(F \circ G)(\bar{x}, \bar{z}) \leq \max _{\bar{y} \in S(\bar{x}, \bar{z})} \operatorname{reg} G(\bar{x}, \bar{y}) \cdot \operatorname{reg} F(\bar{y}, \bar{z}), \\
& \operatorname{cov}(F \circ G)(\bar{x}, \bar{z}) \geq \min _{\bar{y} \in S(\bar{x}, \bar{z})} \operatorname{cov} G(\bar{x}, \bar{y}) \cdot \operatorname{cov} F(\bar{y}, \bar{z}) .
\end{aligned}
$$

Proof. It follows from Theorem 4.2 due to the identity $(F \circ G)^{-1}=G^{-1} \circ F^{-1}$ and the equivalence between the Lipschitz-like and metric regularity/covering properties with the exact bound relationships (2.9) discussed in Section 2.

\section{Marginal Functions}

The concluding section of the paper is devoted to studying the local Lipschitzian property

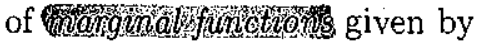

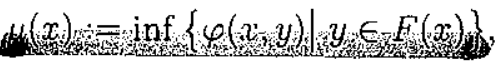


where $\varphi: X \times Y \rightarrow \overline{\mathbb{R}}$ is a l.s.c. cost function and where $F: X \rightrightarrows Y$ is a generating setvalued mapping. We have discussed in Section 1 the meaning and scope of applications of marginal/value functions in variational analysis, optimization, and control.

In this section we develop a subdifferential approach to Lipschitzian stability of marginal functions based on its characterization established in Theorem 2.1(ii). The latter result characterizes the locil Lipschitz continuity of (5.1) around $\bar{x}$ via the simultaneous fulfillment of the singular subdifferential condition $\partial^{\infty} \mu(\bar{x})=\{0\}$ and the SNEC property of $\mu$ at $\bar{x}$, provided that $\mu$ is $1 . s$. around $\bar{x}$ and that $X$ is Asplund. First recall the following convenient subdiffercutal description of the SNEC property for l.s.c. functions on Asplund spaces derived by Mordukhorich and Nam [19].

Lemma 5.1 (subdifferential characterization of SNEC functions). Let $X$ be Asplund, and let $\varphi: X \cdots \bar{R}$ br an crtended-real-valued function that is l.s.c. around $\bar{x}$. Then $\varphi$ enjoys the SNEC proprty at $\bar{x}$ if and only if for any sequences $x_{k} \rightarrow \bar{x}, \lambda_{k} \downarrow 0$, and $x_{k}^{*} \in \lambda_{k} \widehat{\partial} \varphi\left(x_{k}\right)$ as $k \in \Lambda^{*}$ one has the implication.

$$
\left[x_{k}^{*} \stackrel{u}{*}_{i} 0\left[\left\|x_{k}^{*}\right\| \rightarrow 0\right] \text { as } k \rightarrow \infty\right.
$$

Our first goal is to establish a relationship between the singular subdifferential of the marginal function $\mu$ and the mixel coderivative of the generating mapping $F$ in (5.1). To proceed, define some modifications of the inner semicontinuity and inner semicompactness notions from Section 3, which are more stitable for st udying marginal functions.

Given a set-valued mapping $S: X \Rightarrow Y$ between Banach spaces and a function $\mu: X \rightarrow$ $\bar{R}$, we say that $S$ is $\mu$-inner semrontmuous at $(\bar{x}, \bar{y}) \in \operatorname{gph} S$ if for any sequences $\varepsilon_{k} \downarrow 0$ and $x_{k} \stackrel{\mu}{\rightarrow} \bar{x}$ with $\widehat{\partial}_{\varepsilon_{k}} \mu\left(x_{k}\right) \neq \emptyset$ there is a sequence $y_{k} \in S\left(x_{k}\right)$ that contains a subsequence converging to $\bar{y}$. The mapping $S$ is said to be $\mu$-inner semicompact at $\bar{x}$ with $S(\bar{x}) \neq \emptyset$ if for any sequences $\varepsilon_{k} \downarrow 0$ and $x_{k} \stackrel{\mu}{\rightarrow}$ with $\widehat{\partial}_{\Sigma_{h}} \mu\left(x_{k}\right) \neq \emptyset$ there is a sequence $y_{k} \in S\left(x_{k}\right)$ containing a convergent subsequence. Observe as ustal that we can equivalently put $\varepsilon_{k}=0$ if both spaces $X$ and $Y$ are Asplund and $\mu$ is lower semicontinuous around $\bar{x}$.

The following theoren essent jally innroves the previonsly known result by Mordukhovich and Shao [21] that established relationships hetwe'n the singular subdifferential of the marginal function $\mu$ and the normul cokeriwative of the generating mapping in (5.1) under the inner/lower semicompactness assunution on the solution map.

Theorem 5.2 (singular subgradients of marginal functions via mixed coderivatives). Let $F: X \rightrightarrows Y$ be a closed-graph mapping between Asplund spaces, and let

$$
S(x):=\{y \in F(x) \mid \mu(x)=\varphi(x, y)\}
$$

be the solution/minimum map for the marginal function (5.1). The following hold:

(i) Assume that $S$ is $\mu$-inner semicontinuous at $(\bar{x}, \bar{y}) \in \operatorname{gph} S$ and that $\varphi$ is locally Lipschitzian around this point. Then

$$
\partial^{\infty} \mu(\bar{x}) \subset D_{M}^{*} F(\bar{x}, \bar{y})(0)
$$


(ii) Assume that $S$ is $\mu$-inner semicompact at $\bar{x}$ and that $\varphi$ is locally Lipschitzian around $(\bar{x}, \bar{y})$ for all $\bar{y} \in S(\bar{x})$. Then

$$
\partial^{\infty} \mu(\bar{x}) \subset \bigcup_{\bar{y} \in S(\bar{x})} D_{M}^{*} F(\bar{x}, \bar{y})(0) .
$$

Proof. To justify (5.3), fix any singular subgradient $x^{*} \in \partial^{\infty} \mu(\bar{x})$ and get by definition (2.5) that there are sequences

$$
\varepsilon_{k} \downarrow 0, \lambda_{k} \downarrow 0, x_{k} \stackrel{\mu}{\rightarrow} \tilde{x}, \text { and } x_{k}^{*} \in \widehat{\partial}_{\varepsilon_{k}} \mu\left(x_{k}\right)
$$

such that $\lambda_{k} x_{k}^{*} \stackrel{w^{*}}{\longrightarrow} x^{*}$ as $k \rightarrow \infty$. Since $S$ is $\mu$-inner semicontinuous at $(\bar{x}, \bar{y})$, we find $y_{k} \in S\left(x_{k}\right)$ whose subsequence, with no relabeling, converges to $\tilde{y}$. It follows by definition from $x_{k}^{*} \in \widehat{\partial}_{\varepsilon_{k}} \mu\left(x_{k}\right)$ that for any $\eta>0$ there is $\gamma>0$ such that

$$
\left\langle x_{k}^{*}, x-x_{k}\right\rangle \leq \mu(x)-\mu\left(x_{k}\right)+\left(\varepsilon_{k}+\eta\right)\left\|x-x_{k}\right\| \text { whenever } x \in x_{k}+\gamma \mathbb{B} .
$$

Considering the function

$$
\phi(x, y):=\varphi(x, y)+\delta((x, y) ; \operatorname{gph} F)
$$

we easily conclude that

$$
\left\langle\left(x_{k}^{*}, 0\right),\left(x-x_{k}, y-y_{k}\right)\right\rangle \leq \phi(x, y)-\phi\left(x_{k}, y_{k}\right)+\left(\varepsilon_{k}+\eta\right)\left(\left\|x-x_{k}\right\|+\left\|y-y_{k}\right\|\right)
$$

whenever $(x, y) \in\left(x_{k}, y_{k}\right)+\gamma \mathbb{B}$, which gives $\left(x_{k}^{*}, 0\right) \in \widehat{\partial}_{\varepsilon_{k}} \phi\left(x_{k}, y_{k}\right)$.

Fix now an arbitrary sequence $\eta_{k} \downarrow 0$. Since $\varphi$ is locally Lipschitzian around $(\bar{x}, \bar{y})$ while $X$ and $Y$ are Asplund, we apply the well-known fuzzy sum rule for $\varepsilon$-subgradients of $\phi$ (which follows from Theorem 3.1) and find sequences

$$
\begin{aligned}
& \left(x_{1 k}, y_{1 k}\right) \stackrel{\varphi}{\rightarrow}(\bar{x}, \bar{y}),\left(x_{2 k}, y_{2 k}\right) \stackrel{\operatorname{gph} F}{\rightarrow}(\bar{x}, \bar{y}), \\
& \left(x_{1 k}^{*}, y_{1 k}^{*}\right) \in \widehat{\partial} \varphi\left(x_{1 k}, y_{1 k}\right) . \text { and }\left(x_{2 k}^{*}, y_{2 k}^{*}\right) \in \widehat{N}\left(\left(x_{2 k}, y_{2 k}\right) ; \operatorname{gph} F\right)
\end{aligned}
$$

satisfying the estimate

$$
\left\|\left(x_{k}^{*}, 0\right)-\left(x_{1 k}^{*}, y_{1 k}^{*}\right)-\left(x_{2 k}^{*}, y_{2 k}^{*}\right)\right\| \leq \varepsilon_{k}+\eta_{k}
$$

or, equivalently, the following ones:

$$
\left\|x_{k}^{*}-x_{1 k}^{*}-x_{2 k}^{*}\right\| \leq \varepsilon_{k}+\eta_{k} \text { and }\left\|y_{1 k}^{*}+y_{2 k}^{*}\right\| \leq \varepsilon_{k}+\eta_{k} .
$$

Let $\ell>0$ be a Lipschitz constant for $\varphi$ around $(\bar{x}, \bar{y})$. Then $\left\|\left(x_{1 k}^{*}, y_{1 k}^{*}\right)\right\| \leq \ell$, which implies that $\lambda_{k}\left\|\left(x_{1 k}^{*}, y_{1 k}^{*}\right)\right\| \rightarrow 0$ as $k \rightarrow \infty$. By (5.4) we therefore have

$$
\lambda_{k}\left\|y_{2 k}^{*}\right\| \rightarrow 0 \text { and } \lambda_{k} x_{2 k}^{*} \stackrel{w^{*}}{\longrightarrow} x^{*} \text { as } k \rightarrow \infty \text {. }
$$

Taking into account that

$$
\lambda_{k}\left(x_{2 k}^{*}, y_{2 k}^{*}\right) \in \widehat{N}\left(\left(x_{2 k}, y_{2 k}\right) ; \operatorname{gph} F\right) \text { for all } k \in \mathbb{N}
$$


and using the definition of the mixed coderivative (2.7), we derive from the convergence relations (5.5) that $x^{*} \in D_{M}^{*} F(\bar{x}, \bar{y})(0)$, which gives (5.3) and completes the proof of (i). The proof of assertion (ii) is similar with using the $\mu$-inner semicontinuity condition for $S$ instead of the $\mu$-inner semicompactness one in (i).

Combining now the results obtained in Lemma 5.1 and Theorem 5.2, we establish refined sufficient conditions for the local Lipschitz continuity of marginal functions.

Theorem 5.3 (Lipschitz continuity of marginal functions). Let $F: X \Rightarrow Y$ be a closed-graph mapping between Asplund spaces generating the marginal function $\mu$ in (5.1). The following assertions hold:

(i) Assume that the solution map $S$ in (5.2) is $\mu$-inner semicontinuous at $(\bar{x}, \bar{y})$ and that the cost function $\varphi$ is locally Lipschitzian around this point. Then $\mu$ is Lipschitz continuous around $\bar{x}$ provided that it is l.s.c. around $\bar{x}$ and that $F$ is Lipschitz-like around $(\bar{x}, \bar{y})$.

(ii) Assume that $S$ is $\mu$-inner semicompact at $\bar{x}$ and that $\varphi$ is locally Lipschitzian around $(\bar{x}, \bar{y})$ for every $\bar{y} \in S(\bar{x})$. Then $\mu$ is Lipschitz continuous around $\bar{x}$ provided that it is l.s.c. around this point and that $F$ is Lipschitz-like around $(\bar{x}, \bar{y})$ as $\bar{y} \in S(\bar{x})$.

Proof. It is sufficient to justify (i): the proof of (ii) is similar. We have $D_{M}^{*} F(\bar{x}, \bar{y})(0)=\{0\}$ by Theorem 2.1(ii), since $F$ is assumed to be Lipschitz-like around $(\bar{x}, \bar{y})$. Thus $\partial^{\infty} \mu(\bar{x})=$ $\{0\}$ by Theorem 5.2. By the converse statement of Theorem 2.1(ii), it remains checking that $\mu$ is SNEC at $\bar{x}$ to ensure the Lipschitz continuity of $\mu$ around this point. Employing the SNEC characterization from Lemma 5.1 . we take any sequences $\lambda_{k} \downarrow 0, x_{k} \stackrel{\leftrightarrow}{\rightarrow} \bar{x}$, and $x_{k}^{*} \in \lambda_{k} \widehat{\partial} \mu\left(x_{k}\right)$ such that $x_{k}^{*} \stackrel{u^{*}}{\rightarrow} 0$ is $k \rightarrow x$. To finish the proof, we need showing that $\left\|x_{k}^{*}\right\| \rightarrow 0$ along some subsequence of $k \rightarrow x$.

To proceed, use the $\mu$-semicontinuity of $S$ from $(5.2)$ at $(\bar{x}, \bar{y})$ and select a sequence of $y_{k} \in S\left(x_{k}\right)$ whose subsequence converge's (with no relabeling) to $\bar{y}$. Take $\widetilde{x}_{k}^{*} \in \widehat{\partial} \mu\left(x_{k}\right) \leq \ldots$ is that $x_{k}^{*}=\lambda_{k} \widetilde{x}_{k}^{*}$. Similarly to the proof of Theorem 5.2. find sequences

$$
\begin{aligned}
& \left(x_{1 k}, y_{1 k}\right) \stackrel{\dot{\varphi}}{\longrightarrow}(\bar{x}, \bar{y}) . \quad\left(x_{2 k}, y_{2 k}\right) \stackrel{\mathrm{gph} F}{\longrightarrow}(\bar{x}, \bar{y}) . \\
& \left(x_{1 k}^{*}, y_{1 k}^{*}\right) \in \hat{\partial}_{\varphi}\left(x_{1 k}, y_{1 k}\right) \text {. and }\left(x_{2 k}^{*}, y_{2 k}^{*}\right) \in \hat{N}\left(\left(x_{2 k}, y_{2 k}\right) ; \operatorname{gph} F\right)
\end{aligned}
$$

such that $\lambda_{k}\left\|\left(x_{1 k}^{*}, y_{1 k}^{*}\right)\right\| \rightarrow(0.0)$ with the estimates

$$
\left\|\tilde{x}_{k}^{*}-x_{1 k}^{*}-x_{2 k}^{*}\right\| \leq \varepsilon_{k} \text { and }\left\|y_{1 k}^{*}+y_{2 k}^{*}\right\| \leq \varepsilon_{k} \text { as } k \in \mathbb{N} .
$$

This implies that $\lambda_{k}\left\|y_{2 k}^{*}\right\| \rightarrow 0$ as $k \rightarrow \infty$. Taking now into account that

$$
\left(\lambda_{k} x_{2 k}^{*}, \lambda_{k} y_{2 k}^{*}\right) \in \widehat{N}\left(\left(x_{2 k}, y_{2 k}\right): g_{1} h F\right) \Longleftrightarrow \lambda_{k} x_{2 k}^{*} \in \widehat{D}^{*} F\left(x_{2 k}, y_{2 k}\right)\left(-\lambda_{k} y_{2 k}^{*}\right)
$$

and that $F$ is Lipschitz-like around $(\bar{x}, \bar{y})$ with some modulus $\ell>0$, we get from the coderivative estimate for Lipschitz-like mappings (see, c.g., [18, Theorem 1.43]) that

$$
\left\|\lambda_{k} x_{2 k}^{*}\right\| \leq \ell\left\|\lambda_{k} y_{2 k}^{*}\right\| \text { for large } k \in \mathbb{N}
$$


which implies that $\lambda_{k}\left\|x_{2 k}^{*}\right\| \rightarrow 0$. Combining the latter with (5.6) and with $x_{k}^{*}=\lambda_{k} \tilde{x}_{k}^{*}$, we conclude that $\left\|x_{k}^{*}\right\| \rightarrow 0$ as $k \rightarrow \infty$. This justifies the SNEC property of $\mu$ at $\bar{x}$ and thus completes the proof of the theorem.

It is not hard to (heck that the 1.s.c. property of the marginal function assumed in Theorem 5.3 automalically holds if the $\mu$-inner semicontinuity/semicompactness condition on $S$ is imposed arouth the reference points.

In some applicatintw one needs to consider a more general version of the marginal function (5.1) given $i t_{i} 1 \mathrm{l}_{1}$ form

$$
\mu(x, y):=\inf \{\varphi(y, z) \mid z \in F(x)\}
$$

with $\varphi: Y \times Z \rightarrow \overline{\mathbb{R}}$ iukl $F: X=Z$. This particularly covers the class of distance functions to varying/moving sctu defined by.

$$
\mu(x, y):=\inf \{\|y-z\| \mid z \in F(x)\}
$$

whose subdifferential and Lipschit zian properties have been recently studied by Mordukhovich and Nam [19] at both in-set and unt-of-set points: see also the references therein. Now we present the corresponding results for (5.7) obtained from Theorems 5.2 and 5.3. For brevity, consider only the "inner semicontintuls" version of these theorems.

Corollary 5.4 (singular subgradients and Lipschitzian continuity of generalized marginal functions). Let $\xi: Y \times Z \rightarrow \overline{\mathbb{R}}$ and $F: X \rightrightarrows Z$ in the Asplund spaces setting, and let the solution map

$$
S(x, y):=\{z \in F(x) \mid \mu(x, y)=\varphi(y, z)\}
$$

be $\mu$-inner semicontinuous at some point $((\bar{x}, \bar{y}), \bar{z})$. Assume also that $\varphi$ is locally Lipschitzian around $(\tilde{y}, \bar{z})$ and that $F$ is closcd-graph around $(\bar{x}, \bar{z})$. Then

$$
\partial^{\infty} \mu(\bar{x}, \bar{y}) \subset\left\{\left(r^{*}, 0\right) \mid r^{*} \in D_{i}^{*} F(\bar{x}, \bar{z})(0)\right\} .
$$

Moreover, $\mu$ is Lipschitz-contimuous around $(\bar{r}, \bar{y})$ if $\|$ is l.s.c. around this point while $F$ is Lipschitz-like around $(\bar{x}, \bar{\Sigma})$.

Proof. Put $u:=(x, y)$ and define

$$
\tilde{F}(u)=\tilde{F}(x, y):=F(x) . \quad \tilde{F}(u, z):=\tilde{\varphi}(y, z) .
$$

Then we have the representation

$$
\mu(x, y)=\mu(u)=\inf \{\tilde{\varphi}(u, z) \mid z \in \tilde{F}(u)\} .
$$

Applying Theorem 5.2 with $(\bar{x}, \bar{y})$ replaced by $(\bar{u}, \bar{z})=(\bar{x}, \bar{y}, \bar{z})$, we get

$$
\partial^{\infty} \mu(\bar{x}, \bar{y})=\partial^{\infty} \mu(\bar{u}) \subset D_{M}^{*} \tilde{F}((\bar{x}, \bar{y}), \bar{z})(0) .
$$


It is easy to observe the inclusion

$$
D_{M}^{*} \tilde{F}((\bar{x}, \bar{y}), \bar{z})(0) \subset\left\{\left(x^{*}, 0\right) \mid x^{*} \in D_{M}^{*} F(\bar{x}, \bar{z})(0)\right\} .
$$

Summarizing all the above, we arrive at

$$
\partial^{\infty} \mu(\bar{x}, \bar{y}) \subset\left\{\left(x^{*}, 0\right) \mid x^{*} \in D_{M}^{*} F(\bar{x}, \bar{z})(0)\right\} .
$$

The conclusion about the Lipschitz continuity of $\mu$ around $(\bar{x}, \bar{y})$ follows directly from Theorem 5.3 applied to $(5.5)$.

Observe that the generating map $F(\cdot)$ in $(5.1)$, and similarly in (5.7), describes the sets of feasible solutions to the parametric optimization problem:

$$
\text { minimize } \varphi(x, y) \text { subject to } y \in F(x) \text {. }
$$

A general framework for describing feasible sets $F(x)$ in most applied problems is as follows:

$$
F(x):=\{y \in Y \mid g(x, y) \in \Lambda, \quad(x, y) \in \Omega\},
$$

where $g: X \times Y \rightarrow Z, \Lambda \subset Z$, and $\Omega \subset X \times Y$. Systems of type (5.10) are usually called parametric constraint systems. In particular, model (5.10) can be treated as an extension of the feasible solution sets in parametric problems of nonlinear programming with equality and inequality constraints given by

$$
F(x)=\left\{y \in Y \mid \begin{array}{ll}
\varphi_{i}(x, y) \leq 0 \text { for } i=1, \ldots, m, \\
\left.\varphi_{i}(x, y)=0 \text { for } i=m+1, \ldots, m+r\right\} .
\end{array}\right.
$$

Another important special case of $(5.10)$ is

$$
F(x)=\{y \in Y \mid g(x, y)=0\},
$$

which describes implicit functions/multifunctions.

Furthermore, the general framework of (5.1) is useful for modeling sets of optimal solutions to lower-level optimization problems and also solution sets arising from parametric complementarity conditions and variational inequalities. In such cases, the sets $F(x)$ are usually given in the form of parametric generalized equations (or variational conditions) in the sense of Robinson [28]:

$$
F(x)=\{y \in Y \mid 0 \in f(x, y)+Q(x, y)\}
$$

with $f: X \times Y \rightarrow Z$ and $Q: X \times Y \Rightarrow Z$. When. in particular, $Q(y)=N(y ; \Omega)$ is the normal cone mapping to a convex set, model (5.11) describes solution maps to the classical parameterized variational inequalities.

Having this in mind, one may treat the marginal function (5.1) with the generating mapping $F(x)$ given in form (5.11) as the value function in a mathematical program with equilibrium constraints (MPEC); see Facchinei and Pang [6] for more examples and discussions. Thus, to make a conclusion on Lipschitz stability/continuity of value functions in parametric optimization problems of type (5.9) with constraint sets (5.10) and/or in 
MPECs and related problems with constraints sets of type (5.11), we actually need-by Theorem 5.3-to check the Lipschitz-like property of mappings $F(x)$ given by $(5.10)$ and (5.11). Concerning the latter issue, we refer the reader to Chapter 4 of the book by Mordukhovich [18], which contains calculations/estimates of coderivatives for systems $(5.10)$, (5.11), and their specifications together with verifiable conditions for their Lipschitzian stability in terms of the initial data in both finite-dimensional and infinite-dimensional settings.

Acknowledgments. This research was partially supported by the National Science Foundation under grant I).1S-()304989 and by the Australian Research Council under grant DP-0451168.

\section{References}

[1] Aubin, J.-P. 1984. L.1pwith\% Bedawion of solutions to convex minimization problems. Math. Oper. Res. 9 87-111.

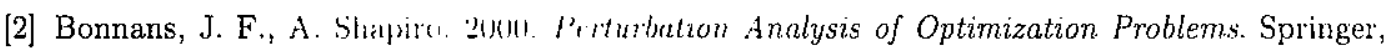
New York.

[3] Borwein, J. M., Q. J. Zhuı. Techmques of l'uriational Analysis. An Introduction. Springer, New York, to appear.

[4] Clarke, F. H., Yu. S. Ledyaer. R. J. Stem. P. R. Wolenski. 1998. Nonsmooth Analysis and Control Theory. Springer. New York.

[5] Dontchev, A. L., A. S. Lewis. R. T. Rockafellar. The radius of metric regularity. Trans. Amer. Math. Soc. 355 493-jli.

[6] Facchinei, F., J.-S. Pang. 2003. Finte-Dimensional l'ariational Inequalities and Complementarity Problems, Volumes I and II. Springer. New York.

[7] Ioffe, A. D. 2000. Codirectional compactness. metric regularity and subdifferential calculus.: M. Théra, ed. Constructile. Expermmental and Nonlmerar Analysis. American Mathematical Society, Providence. RI. 123 16:4.

[8] Ioffe, A. D., J.-P. Penot. 1996 . Subliffirentinals of performance functions and calculus of

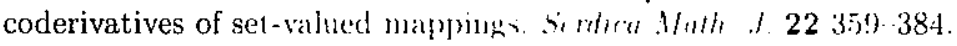

[9] Jourani, A., L. Thibauh. 1999. Qnatificancin comulnum ion calculus rules of coderivatives of multivalued mappings. J. Wath Ant ilpht 218 (iti $: 1$.

[10] Jourani, A., L. Thibault. 1999. Coderivatives of muluwalued mappings, locally compact cones and metric regularity. Nonlinear Amal. 35 925 945 .

[11] Levy, A. B., B. S. Mordukhovich. 2004. Coderivatives in paranetric optimization, Math. Progr., Ser. A 99 311-327.

[12] Mordukhovich, B. S. 1976. Maxinum principle in problems of time optimal control with nonsmooth constraints. J. Appl. Math. Mech. 40 960-969.

[13] Mordukhovich, B. S. 1980. Metric approximations and necessary optimality conditions for general classes of nonsmooth extremal problnies. Soviet Math. Dokl. 40 526-530.

[14] Mordukhovich, B. S. 1993. Complete characterizations of openness, metric regularity and Lipschitzian properties of muitifunctions. Trans. Amer. Math. Soc. 340 1-36. 
[15] Mordukhovich, B. S. 1994. Generalized differential calculus for nonsmooth and set-valued mappings. J. Math. Anal. Appl. 183 250-288.

[16] Mordukhovich, B. S. 1997. Coderivatives of set-valued mappings: calculus and applications. Nonlinear Anal. 30 3059-3070.

[17] Mordukhovich, B. S. 2004. Coderivative analysis of variational systems. J. Global Optim. 28 347-362.

[18] Mordukhovich, B. S. Variational Analysis and Generalized Differentiation, Vol. I: Basic Theory, Vol. II: Applications. Springer, Berlin, to appear.

[19] Mordukhovich, B. S.. N. M. Nam. 2004. Subgradiens of distance functions with applications to Lipschitzian stability. Math. Progr. Ser. B, to appear.

[20] Mordukhovich, B. S.. Y. Shao. 1996a. Nonsmooth sequential analysis in Asplund spaces. Trans. Amer. Math. Soc. 348 1235-1280.

[21] Mordukhovich, B. S.. Y. Shao. 1996b. Nonconvex differential calculus for infinite-dimensional multifunctions. Set-Vialued Anal. 4 205-236.

[22] Mordukhovich, B. S. Y. Shao. 1997. Fuzzy calculus for coderivatives of multifunctions. Nonlinear Anal. 29 605-626.

[23] Mordukhovich, B. S., Y. Shao. 1998. Mixed coderivatives of set-valued mappings in variational analysis. J. Appl. Anal. 4 269-294.

[24] Mordukhovich, B. S., B. Wang. 2003. Calculus of sequential normal compactness in variational analysis, J. Math. Anal. Appl. 282 63-84.

[25] Outrata, J. V. 1999. Optimality conditions for a class of mathematical programs with equilibrium constraints. Math. Oper. Res. 24 627-644.

[26] Penot, J.-P. 1998. Compactness properties, openness criteria and coderivatives. Set-Valued Anal. $6363-380$.

[27] Phelps, R. R. 1993. Convex Functions, Monotone Operators and Differentiability, 2nd ed. Springer, Berlin.

[28] Robinson, S. M. 2003. Constraint nondegeneracy in variational analysis. Math. Oper. Res. 28 201-232.

[29] Rockafellar, R. T. 1981. Proximal subgradients, marginal values and augmented Lagrangians in nonconvex optimization. Math. Oper. Res. 6 424-436

[30] Rockafellar, R. T., R. J-B. Wets. 1998. Variational Analysis. Springer, Berlin.

[31] Thibault, L. 1980. Subdifferentials of compactiy Lipschitzian vector-valued functions. Ann. Mat. Pura Appl. 125 157-192.

[32] Thibault, L. 1991. On subdifferentials of optimal value functions. SIAM J. Control Optim. 29 1019-1036.

[33] Treiman, J. S. 1999. Lagrange multipliers for nonconvex generalized gradients with equality, inequality, and set constraints. SIAM J. Control Optim. 37 1313-1329.

[34] Vinter, R. B. 2000. Optimal Control. Birkhäuser, Boston.

[35] Ye, J. J., X. Y. Ye. 1997. Necessary optimality conditions for optimization problems with variational inequality constraints. Math. Oper. Res. 22 977-997.

[36] Ye, J. J., Q. J. Zhu. 2003. Multiobjective optimization problems with variational inequality constraints. Math. Progr., Ser. A 96 139-160. 